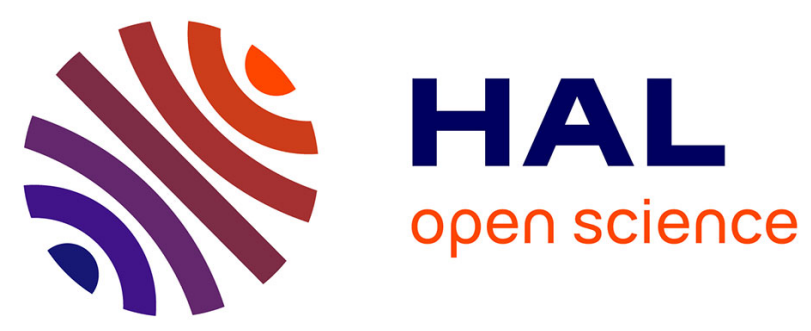

\title{
Investigating the cross-slip rate in face-centered cubic metals using an atomistic-based cross-slip model in dislocation dynamics simulations
}

\author{
M. Longsworth, Fivel Marc
}

\section{- To cite this version:}

M. Longsworth, Fivel Marc. Investigating the cross-slip rate in face-centered cubic metals using an atomistic-based cross-slip model in dislocation dynamics simulations. Journal of the Mechanics and Physics of Solids, 2021, 153 (2), pp.104449. 10.1016/j.jmps.2021.104449 hal-03219945

\section{HAL Id: hal-03219945 \\ https://hal.science/hal-03219945}

Submitted on 6 May 2021

HAL is a multi-disciplinary open access archive for the deposit and dissemination of scientific research documents, whether they are published or not. The documents may come from teaching and research institutions in France or abroad, or from public or private research centers.
L'archive ouverte pluridisciplinaire HAL, est destinée au dépôt et à la diffusion de documents scientifiques de niveau recherche, publiés ou non, émanant des établissements d'enseignement et de recherche français ou étrangers, des laboratoires publics ou privés. 


\section{Graphical Abstract}

Investigating the cross-slip rate in face-centered cubic metals using an atomistic-based cross-slip model in dislocation dynamics simulations

M. Longsworth, M. Fivel

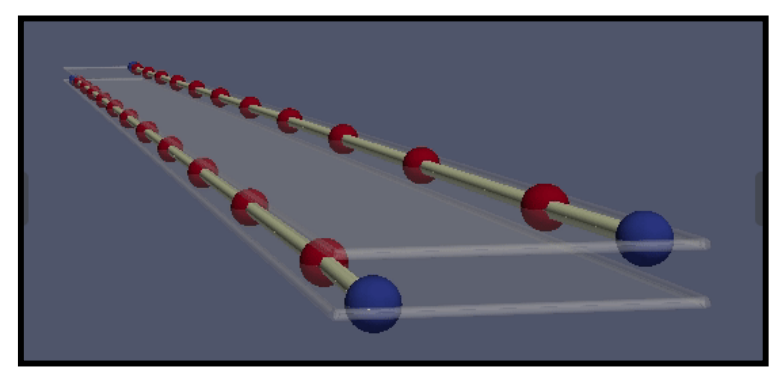

1. Attractive screw dipole.

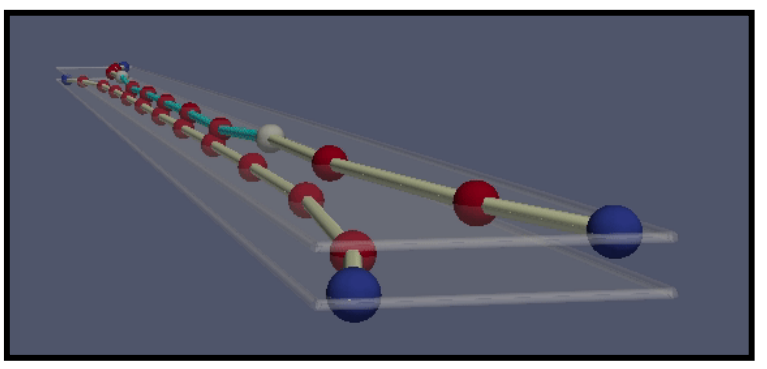

4. If (green) segment cross-slips, it glides in the conjugate plane.

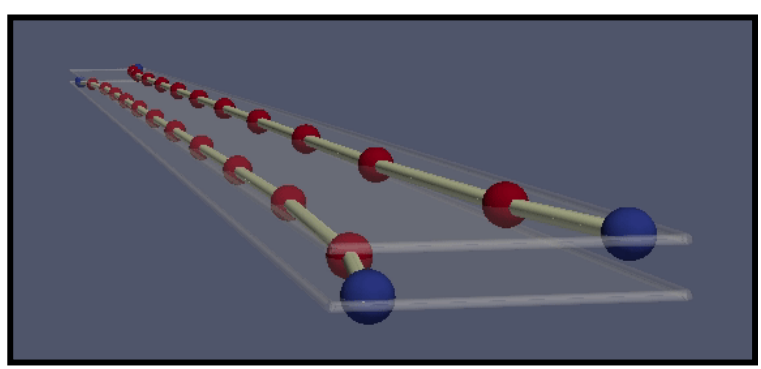

2. Identify screw segments.

3. Evaluate atomic-based cross-slip probability of screw segments.

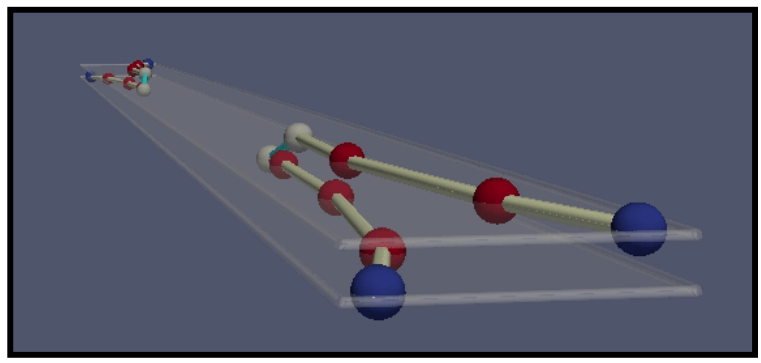

5. Both dislocations annihilate each other. 


\section{Highlights}

Investigating the cross-slip rate in face-centered cubic metals using an atomistic-based cross-slip model in dislocation dynamics simulations

M. Longsworth, M. Fivel

- Cross-slip rate model in DD simulations reproduces Arrhenius plots.

- Cross-slip energy model in DD simulations reproduces atomistic results.

- No scaling factors used; only unstressed energy barrier is required.

- Unstressed energy barrier used obtained from DD simulations. 


\title{
Investigating the cross-slip rate in face-centered cubic metals using an atomistic-based cross-slip model in dislocation dynamics simulations
}

\author{
M. Longsworth, M. Fivel \\ Univ. Grenoble Alpes, CNRS, Grenoble INP, SIMaP, Grenoble 38000, France
}

\begin{abstract}
The cross-slip rate of screw segments in dislocation dynamics simulations was calculated using the model of Esteban-Manzanares et al. (2020), which is based on a combination of the harmonic transition state theory and the Meyer-Neldel rule. In said model, the cross-slip rate is expressed as a function of the microstructure parameters. In particular, the rate prefactor depends on the nucleation length of cross-slip and the activation enthalpy, which are themselves functions of the local stress. Malka-Markovitz and Mordehai (2019) solved the line tension model of cross-slip exactly by linearizing the interaction force between the partials. They obtained analytical expressions for the nucleation length and the activation enthalpy as functions of a general stress state. These expressions were used to evaluate the cross-slip rate at each simulation step. The results are in quantitative agreement with atomistic simulations.
\end{abstract}

Keywords:

cross-slip rate, cross-slip enthalpy, dislocation dynamics, line tension model

\section{Introduction}

The cross-slip mechanism is a thermally activated process in which screw dislocations change their glide plane, allowing them to overcome obstacles and populate other slip planes (Anderson et al., 2017). It plays an important role during plastic deformation by creating dislocation structures (Johnston and Gilman, 1960; Ikeno and Furubayashi, 1972), controlling the dynamic recovery (Hirsch, 2009) and producing work hardening (Jackson, 1985; Sudmanns et al., 2019).

Preprint submitted to the Journal of Mechanics and Physics of Solids February 4, 2021 
Many dislocation dynamics (DD) simulations have successfully modeled plastic deformation in crystals (Zbib et al., 1998; Weygand et al., 2002; Verdier et al., 1998; Déprés et al., 2004; Chaussidon et al., 2008). The first cross-slip model used in DD simulations was proposed by Kubin et al. (1992). They postulated that the cross-slip probability explicitly depends on the resolved shear stress at the onset of stage-III plastic deformation, which is a macroscopic parameter obtained from the stress-strain curve of the material:

$$
P=\beta \frac{L}{L_{0}} \exp \left(\frac{\left[\tau-\tau_{I I I}\right] V}{k_{b} T}\right) \delta t
$$

where $\beta$ is a scaling factor, $L$ the screw-segment length, $L_{0}$ a reference length, $\tau$ the resolved shear stress in the primary plane, $\tau_{I I I}$ the resolved shear stress at the onset of stage III, $V$ the activation volume, $k_{b}$ the Boltzmann constant, $T$ the absolute temperature and $\delta t$ the time step.

The values for the activation volume and resolved shear stress at the onset of stage III were obtained from experiments. However, the scaling factor and reference length were defined arbitrarily. In consequence, the absolute crossslip rates were difficult to validate using other simulation methods.

Hussein et al. (2015) proposed a more physical model depending only on the microstructure parameters:

$$
P=\omega \frac{L}{L_{0}} \exp \left(-\frac{\Delta E_{c}-V \Delta \sigma_{E}}{k_{b} T}\right) \delta t
$$

where $\omega$ is the attempt frequency, $\Delta E_{c}$ the energy barrier required to form a constriction point on the screw dislocation, and $\Delta \sigma_{E}=\sigma_{E}^{g}-\sigma_{E}^{c s}$ the difference of Escaig stress on the glide and cross-slip planes.

Although the energy barrier and activation volume were obtained from atomistic simulations, the attempt frequency was determined using an heuristic argument, which introduced uncertainty in the absolute cross-slip rates.

The models 1 and 2 depend either on the Schmid or Escaig stresses, but not on both. Hence, they might be incomplete descriptions of the crossslip rate. In fact, Kang et al. (2014) showed that both stress components have a comparable effect on the activation enthalpy. This assertion has been thoroughly verified using different formulations of the Line Tension (LT) model (Kang et al., 2014; Liu et al., 2019; Longsworth and Fivel, 2021), linear-elasticity models (Longsworth and Fivel, 2021; Kuykendall et al., 2020) and atomistic simulations (Kang et al., 2014; Esteban-Manzanares et al., 
2020; Kuykendall et al., 2020; Liu et al., 2019). Therefore, the influence of all the stress components should be considered in the cross-slip rate.

More recently, Malka-Markovitz et al. (2021) calculated the cross-slip probability of screw segments in DD simulations using the following equation:

$$
P=\beta \frac{L}{L_{0}} \exp \left(-\frac{\Delta H}{k_{b} T}\right) \delta t
$$

where $\Delta H$ is the activation enthalpy.

They considered the full effect of a general stress in the activation enthalpy by using the analytical expression of Malka-Markovitz and Mordehai (2019) to calculate it. Nonetheless, their semi-empirical model of the cross-slip rate requires the scaling factor $\beta$ to be calibrated with atomistic results.

The present work provides a method to calculate the cross-slip rate of screw segments in DD simulations without relying on scaling factors, yet able to reproduce atomistic results.

The rest of the paper is organized as follows. Section 3 introduces the rate equation proposed by Esteban-Manzanares et al. (2020) to compute the cross-slip probability of screw segments; the general expression obtained by Malka-Markovitz and Mordehai (2019) to calculate the activation enthalpy and the DD simulation setup. Section 4 shows a comparison between the annihilation rates of a screw dipole obtained from DD simulations and the atomistic results of Vegge et al. (2000) and Oren et al. (2017) as a reference. Finally, Section 5 presents the summary and conclusions of this work.

\section{Theory}

\subsection{Cross-slip rate equation}

Consider the potential energy $\Phi\left(y_{1}, \ldots, y_{N}\right)$ of a system having only one saddle point $P$ between two minima $A$ and $B$. There is a unique hypersurface $S$ of dimension $N-1$ passing though $P$ that is perpendicular to the level curves of $\Phi$. Let $S_{0}$ be another hyper-surface with the same shape as $S$, but passing through $A$ and oriented such that the normal to $S_{0}$ at $\mathrm{A}$ is along the line of force leading to $P$ (see Fig. 1). In this framework, the following constrained partition functions are defined (Vineyard, 1957):

$$
Q_{S}=\rho_{0} \int_{S} \exp \left(-\frac{\Phi}{k_{b} T}\right) d S
$$




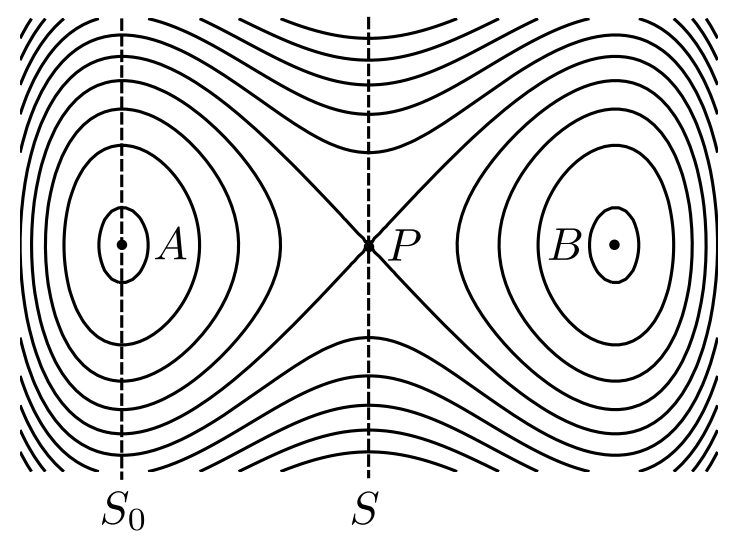

Figure 1: Contour plot of a potential with two minima and a saddle point. The solid lines represent level curves and the segmented ones hyper-surfaces.

$$
Q_{0}=\rho_{0} \int_{S_{0}} \exp \left(-\frac{\Phi}{k_{b} T}\right) d S_{0}
$$

and

$$
Q_{A}=\rho_{0} \int_{A} \exp \left(-\frac{\Phi}{k_{b} T}\right) d V
$$

where $\rho_{0}$ is a normalization factor, $k_{b}$ the Boltzmann constant, $T$ the absolute temperature, $\int_{S}$ the surface integral over $S, \int_{S_{0}}$ the surface integral over $S_{0}$ and $\int_{A}$ the volume integral under $S$.

In Transition State Theory (TST), the rate of transition from state $A$ to $B$ can be expressed as (Vineyard, 1957):

$$
\Gamma_{T S T}=\sqrt{\frac{k_{b} T}{2 \pi}} \frac{Q_{S}}{Q_{A}}
$$

Multiplying and diving the latter equation by $Q_{0}$ :

$$
\Gamma_{T S T}=\sqrt{\frac{k_{b} T}{2 \pi}} \frac{Q_{0}}{Q_{A}} \frac{Q_{S}}{Q_{0}}=\sqrt{\frac{k_{b} T}{2 \pi}} \frac{Q_{0}}{Q_{A}} \exp \left(-\frac{\Delta F}{k_{b} T}\right)
$$

where $\Delta F$ is the free-energy change from $A$ to $P$. The rate prefactor represents the effective frequency $\tilde{\nu}$ : 


$$
\tilde{\nu}=\sqrt{\frac{k_{b} T}{2 \pi}} \frac{Q_{0}}{Q_{A}}
$$

In systems subject to constant stress, $\Delta F$ is the Gibbs free-energy $\Delta G \equiv$ $\Delta H-T \Delta S$, where $\Delta H$ and $\Delta S$ are the activation enthalpy and entropy, respectively. Under this assumption, Eq. 8 can be rewritten as:

$$
\Gamma_{T S T}=\tilde{\nu} \exp \left(\frac{\Delta S}{k_{b}}\right) \exp \left(-\frac{\Delta H}{k_{b} T}\right)
$$

In Harmonic TST (HTST), the potential energy is expanded in Taylor series around $A$ up to second order, which leads to the following simplified rate equation (Vineyard, 1957; Granato et al., 1964):

$$
\Gamma_{H T S T}=\nu_{1} \prod_{i=2}^{N} \frac{\nu_{i}}{\nu_{i}^{\prime}} \exp \left(-\frac{\Delta H}{k_{b} T}\right)
$$

where $\nu_{i}$ are the eigenfrequencies of state $A$ and $\nu_{i}^{\prime}$ those of the transition state $P$. In particular, $\nu_{1}$ is the fundamental frequency.

A comparison between Eqs. 10 and 11 shows that the entropic factor $e^{\Delta S / k_{b}}$ is approximately equal to $\prod_{i=2}^{N} \frac{\nu_{i}}{\nu_{i}^{\prime}}$ if $\tilde{\nu}$ is considered to be $\nu_{1}$.

The activation entropy can also be calculated using an empirical relation known as the Meyer-Neldel (MN) rule (Meyer and Neldel, 1937):

$$
\Delta S=\frac{\Delta H}{T_{m}}
$$

where $T_{m}$ is the melting temperature.

Esteban-Manzanares et al. (2020) studied the effect of stress on the crossslip rate in Aluminum using atomistic simulations. They found a rate equation in great quantitative agreement with their results by applying the MN rule within the HTST framework:

$$
\Gamma_{H T S T}=\nu \frac{L}{L_{n}} \exp \left(-\frac{\Delta H}{k_{b} T}\left[1-\frac{T}{T_{m}}\right]\right)
$$

where $\nu=10^{11} \mathrm{~Hz}$ is the fundamental frequency (Sobie et al., 2017), $L$ the screw-dislocation length and $L_{n}$ the nucleation length of cross-slip. 


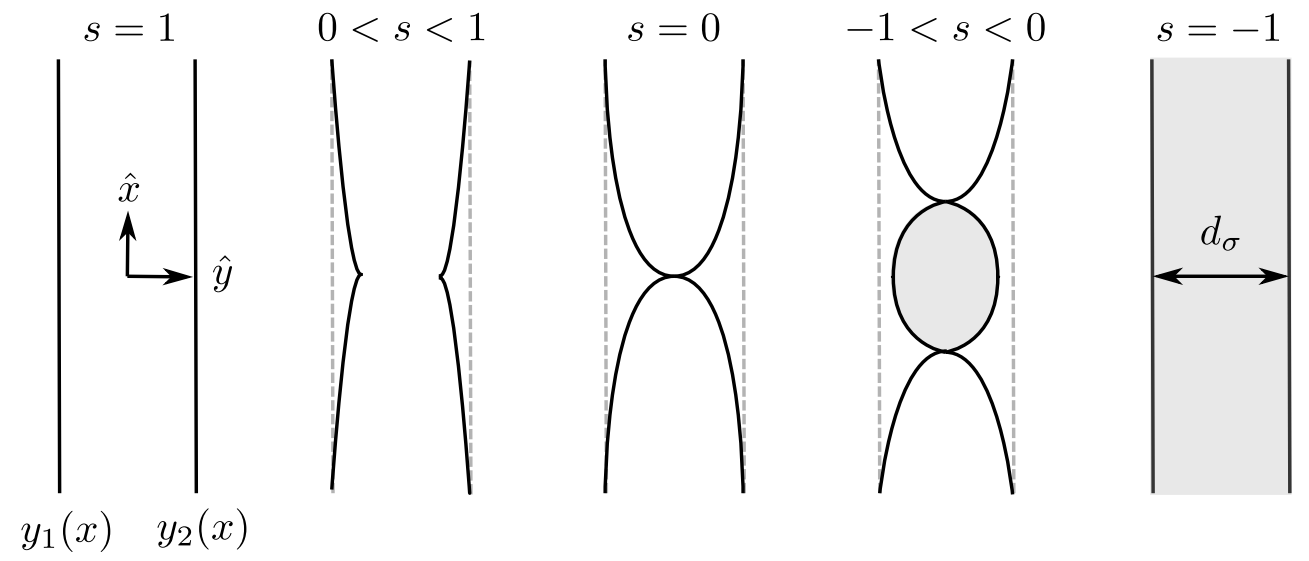

Figure 2: Cross-slip stages of a dissociated screw dislocation as function of the reaction coordinate $s$. The shaded region indicates that the stackingfault is located the cross-slip plane.

Source: Figure adapted from Malka-Markovitz and Mordehai (2018).

\subsection{Nucleation length of cross-slip}

In this study, the nucleation length was inferred from a LT model of cross-slip. According to said model, the dislocations can be approximated as flexible strings with line tension $T_{p}$ (Kang et al., 2014):

$$
T_{p}=\frac{\alpha \mu b^{2}}{2}
$$

where $\alpha$ is the line-energy parameter, $\mu$ is the shear modulus and $b$ the magnitude of the Burgers vector.

The total energy of the dislocations $E_{s}$ is a function of the line tension, the shape of the partials $y_{1}(x)$ and $y_{2}(x)$ and the interaction energy between them $V\left(y_{1}, y_{2}\right)$ (Malka-Markovitz and Mordehai, 2018):

$$
E_{s}=\int_{-\infty}^{+\infty}\left[\frac{1}{2} T_{p}\left(y_{1}^{\prime}\right)^{2}+\frac{1}{2} T_{p}\left(y_{2}^{\prime}\right)^{2}+V\right] d x
$$

The prime symbol denotes the derivative with respect to the line direction $x$. Under Escaig stress $\sigma_{E}$, the equilibrium separation between the partials is given by (Malka-Markovitz and Mordehai, 2018):

$$
d_{\sigma}=\beta\left(\sigma_{E}\right) d_{0}
$$


where:

$$
\beta\left(\sigma_{E}\right)=\frac{1}{1+\frac{\sqrt{3} b}{6 \gamma} \sigma_{E}} \quad \text { and } \quad d_{0}=\frac{\mu b^{2}}{24 \pi \gamma} \frac{2-3 \nu}{1-\nu}
$$

$\gamma$ being the intrinsic stacking-fault energy and $\nu$ the Poisson's ratio. In absence of stress, $\beta(0)=1$ and $d_{\sigma}$ reduces to $d_{0}$ as expected.

The dissociation width $D(x)$ satisfies $D(x)=y_{1}(x)-y_{2}(x)$. Therefore, an infinite dislocation at equilibrium satisfies $D(x)=d_{\sigma}$ for all $x$.

The reaction coordinate $s$ is a dimensionless variable defined in the interval $[-1,1]$. The cross-slip process as function of $s$ is illustrated in Fig. 2. For $s \in(0,1]$, the dislocation is fully contained in the glide plane at equilibrium. The dissociation width at the pinning point $x=0$ decreases as $s$ goes to 0 i.e. $D_{s}(0)=s d_{\sigma}$. At $s=0$, the dislocation constricts, which means that $D_{s}(0)=0$. For $s \in[-1,0)$, the dislocation expands in the cross-slip plane. When only Escaig stresses are applied, the dissociation width at the pinning point can be expressed as $D_{s}(0)=|s| d_{\sigma}$. The cross-slip process finishes when $s=-1$, as the dislocation is completely contained in the conjugate plane at equilibrium.

The energy functional $E_{s}\left(y_{1}, y_{2}\right)$ can be minimized using the Euler-Lagrange equation in order to find the cross-slip trajectory. Applying the boundary conditions $D_{s}(x \rightarrow \infty)=d_{\sigma}$ and $D_{s}^{\prime}(x \rightarrow \infty)=0$, it can be shown that the dissociation width in the glide plane satisfies (Malka-Markovitz and Mordehai, 2018):

$$
x=d_{\sigma} \sqrt{\frac{T_{p}}{4}} \int_{s}^{D_{s}^{*}(x)} \frac{d \eta}{\sqrt{V(\eta)}}
$$

where $D_{s}^{*}=D_{s} / d_{\sigma}$ is the normalized dissociation width between the partials and $\eta$ an adimensional integration variable.

The linear approximation can be used to model the interaction force between the partials for distances near the equilibrium dissociation width $d_{0}$. This is called the Harmonic Approximation (HA) because the resulting interaction energy is a quadratic function of their separation. It can be shown that using this approximation, the interaction potential in the glide plane is given by (Malka-Markovitz and Mordehai, 2018):

$$
V_{H A}^{g}\left(D^{*}\right)=\frac{1}{2}\left(\gamma d_{0}\right)\left(D^{*}-1\right)^{2}
$$


Substituting Eq. 19 into Eq. 18 gives the relation between $x$ and $D^{*}$ :

$$
x=\beta_{g} \sqrt{\frac{T_{p} d_{0}}{2 \gamma}} \int_{s}^{D_{s}^{*}(x)} \frac{d \eta}{|\eta-1|}
$$

The notation $\beta_{g}$ means $\beta\left(\sigma_{E}^{g}\right)$, where $\sigma_{E}^{g}$ is the Escaig stress on the glide plane (see Eq. 17).

Thus, the normalization factor in front of the integral could be interpreted as the dislocation length bowing towards the constriction (Malka-Markovitz and Mordehai, 2018). For that reason, it was used in the present work to estimate the nucleation length of cross-slip $L_{n}$ on the glide plane:

$$
L_{n}=\beta_{g} \sqrt{\frac{T_{p} d_{0}}{2 \gamma}}
$$

Since $\sigma_{E}^{g}$ appears in the denominator of $\beta_{g}, L_{n}$ decreases with positive Escaig stress on the glide plane. In the convention of Malka-Markovitz and Mordehai (2018), a positive Escaig stress shrinks the dissociation width, which increases the cross-slip probability.

\subsection{Activation enthalpy}

The activation enthalpy can be also obtained from the LT model of crossslip. The first formulation of it was proposed by Stroh (1954) to estimate the effect of Escaig stress in the constriction energy. Kang et al. (2014) generalized it by including the influence of Schmid stress in the cross-slip plane. Later on, Malka-Markovitz and Mordehai (2019) generalized Strohs's LT model (Stroh, 1954) for an arbitrary interaction force between the partials, and then solved it analytically by using the linear approximation for the interaction force. Malka-Markovitz and Mordehai (2019) assumed the FE mechanism in their LT model, which implies that the dislocation does not move before cross-slipping. As a consequence, the Schmid stress on the glide plane $\sigma_{S}^{g}$ does not appear in their model. They found a general expression for the cross-slip energy as a function of the separation between the constriction points $l$, the elastic constants and the local stress. In their model, the cross-slip energy reaches its maximum when the separation between the constriction points $l=l_{c}$ satisfies the following critical equation (Malka-Markovitz et al., 2021): 


$$
\frac{1.55}{\cosh ^{2}\left(l_{c}\right)}-\frac{3 \delta}{\beta_{c s}} l_{c}^{2}=2 E^{*}
$$

where $\delta$ is a function of the Schmid stress on the cross-slip plane $\sigma_{S}^{c s}$ and $E^{*}$ is a dimensionless off-set in the interaction energy between the glide and cross-slip planes:

$$
\delta=\frac{1}{6}\left(\frac{b \sigma_{S}^{c s}}{\gamma}\right)^{2} \text { and } E^{*}=\ln \left(\frac{\beta_{c s}}{\beta_{g}}\right)
$$

The notation $\beta_{c s}$ means $\beta\left(\sigma_{E}^{c s}\right)$, where $\sigma_{E}^{c s}$ is the Escaig stress on the cross-slip plane (see Eq. 17). Once the value of $l_{c}$ was found, the activation enthalpy $\Delta H$ is obtained by evaluating the corrected cross-slip energy $H(\sigma, l)$ at $l=l_{c}$ (Malka-Markovitz et al., 2021):

$$
\frac{H(\sigma, l)}{\Delta E_{0}}=\frac{\beta_{g}}{2}+\left(\frac{\beta_{c s}}{2}\right)\left[\tanh (l)-\frac{2 \alpha_{L s}}{1.55} l E^{*}-\frac{\alpha_{L s}^{3}}{1.55} \frac{\delta}{\beta_{c s}} l^{3}\right]
$$

where $\Delta E_{0}$ is the unstressed energy barrier and $\alpha_{L s} \approx 0.6$. In the present work, we consider the energy barrier to be the maximum of Eq. 24. For that reason, the critical equation for $l_{c}$ was obtained by differentiating Eq. 24 with respect to $l$ and equating it to zero, which gives:

$$
\frac{1.55}{\cosh ^{2}\left(l_{c}\right)}-\frac{3 \alpha_{L s}^{3} \delta}{\beta_{c s}} l_{c}^{2}=2 \alpha_{L s} E^{*}
$$

Fig. 3 shows the minor difference between evaluating $H(\sigma, l)$ at the critical length given by Eqs. 22 and 25 for FCC copper. The magnitude of the Burgers vector was assumed to be $b=2.55 \AA$, the intrinsic stacking-fault energy $\gamma=42 \mathrm{~mJ} / \mathrm{m}^{2}$ and the unstressed energy barrier $\Delta E_{0}=1.9 \mathrm{eV}$. Notice that the activation enthalpy obtained by evaluating $H(\sigma, l)$ at the solution of Eq. 22 is slightly lower than the maximum of $H(\sigma, l)$ for a given stress condition.

\section{Methodology}

The cross-slip process in DD simulations is usually modeled using a kinetic Monte Carlo method (Kubin et al., 1992; Hussein et al., 2015; Déprés et al., 
(A)

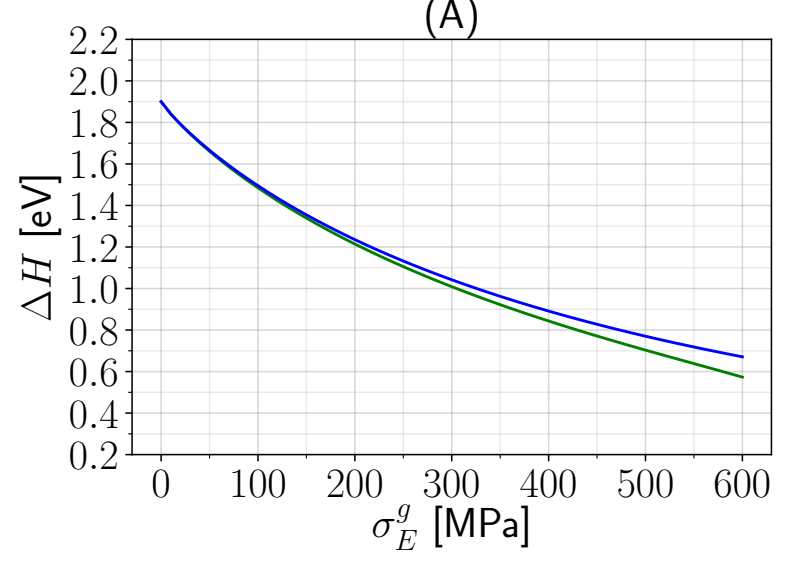

(B)

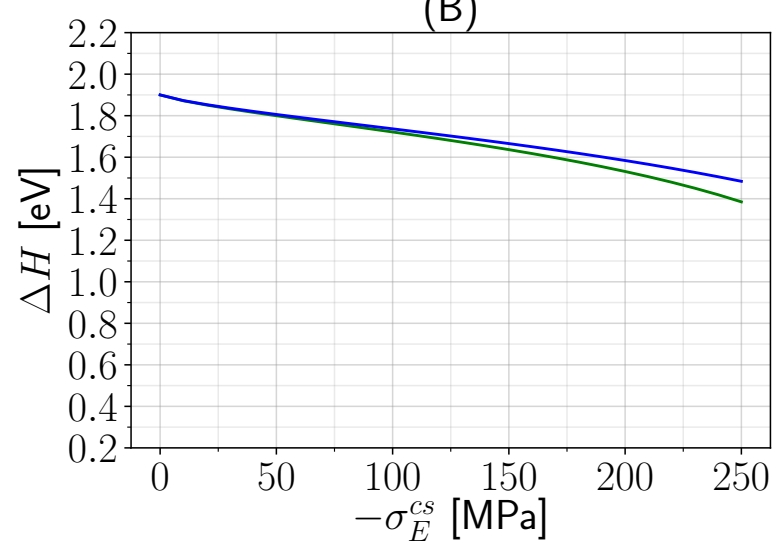

(C)

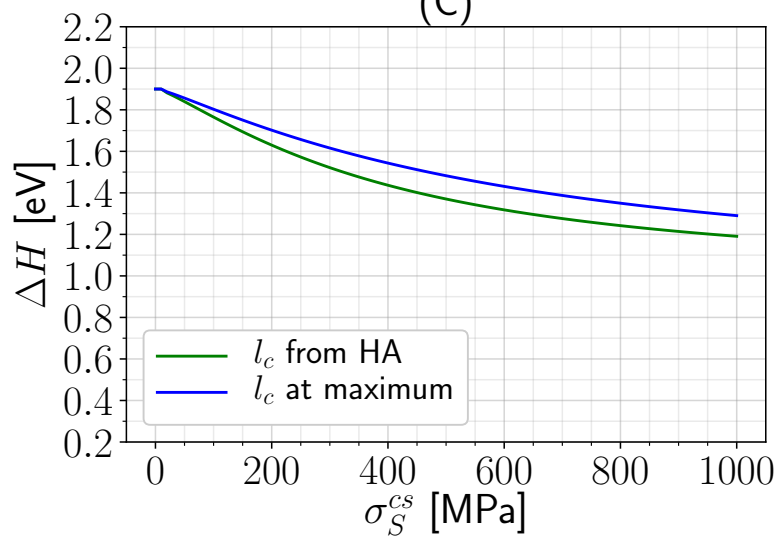

Figure 3: Effect of the stress components on the energy barrier for: (A) compressive Escaig stress on the glide plane $\left(\sigma_{S}^{g}=\sigma_{E}^{c s}=\sigma_{S}^{c s}=0\right)$, (B) expansive Escaig stress on the cross-slip plane $\left(\sigma_{S}^{g}=\sigma_{E}^{g}=\sigma_{S}^{c s}=0\right)$ and (C) Schmid stress on the cross-slip plene $\left(\sigma_{S}^{g}=\sigma_{E}^{g}=\sigma_{E}^{c s}=0\right)$. The green line shows the energy barrier obtained by evaluating $H$ (see Eq. 24) at the solution of Eq. 22. The blue line show the energy barrier obtained by evaluating $H$ at the solution of Eq. 25. 
2004; Malka-Markovitz et al., 2021). In the present work, the cross-slip rate proposed by Esteban-Manzanares et al. (2020) (see Eq. 13) was implemented in the DD code NuMoDis (Drouet et al., 2014) to compute the cross-slip probability of screw segments. Thus, the probability of a screw segment of length $L$ to cross-slip during a time step $\delta t$ is given by:

$$
P=\nu \frac{L}{L_{n}} \exp \left(-\frac{\Delta H}{k_{b} T}\left[1-\frac{T}{T_{m}}\right]\right) \delta t
$$

where $\nu=10^{11} \mathrm{~Hz}$ is the fundamental frequency (Sobie et al., 2017), $L_{n}$ the nucleation length of cross-slip, $k_{b}$ the Boltzmann constant, $T$ the absolute temperature, $T_{m}$ the melting temperature and $\Delta H$ the activation enthalpy.

The activation enthalpy was obtained by evaluating the cross-slip energy $H(\sigma, l)$ (see Eq. 24) at the critical length satisfying Eq. 25. The only free parameter in the theory of Malka-Markovitz and Mordehai (2019) is the unstressed energy barrier $\Delta E_{0}$, which can be obtained either from line tension models (Kang et al., 2014; Malka-Markovitz and Mordehai, 2019), atomistic simulations (Oren et al., 2017; Liu et al., 2019; Esteban-Manzanares et al., 2020; Kuykendall et al., 2020) or DD simulations (Ramírez et al., 2012; Longsworth and Fivel, 2021). In the present paper, the energy barrier of FCC copper $\Delta E_{0}=1.9 \mathrm{eV}$ was obtained from DD simulations (Longsworth and Fivel, 2021) to make the work independent of other numerical results.

The nucleation length was deduced from the theory developed by MalkaMarkovitz and Mordehai (2018) (see Eq. 21). In said model, the only free variable is the line tension $T_{p}$, which is proportional to the line-energy parameter $\alpha$ (see Eq. 14). Typical values of the line-energy parameter are in the range between 0.1 and 0.6. Kang et al. (2014) studied the cross-slip energy of FCC nickel using Molecular Dynamics (MD) simulations and their line tension model. They reported a reasonable agreement between their atomistic simulations and LT model for $\alpha$ values in the range from 0.1 to 0.6 . In a previous work (Longsworth and Fivel, 2021), the line-energy parameter was calibrated using the LT model of Kang et al. (2014). In order to obtain an unstressed energy barrier of $1.9 \mathrm{eV}$ in FCC copper, the line-energy parameter was adjusted to 0.22 . For that reason, an average line-energy value of 0.3 was used in the present work.

The screw chains were identified in the dislocations every simulation step. A dislocation chain was considered to have a screw character if the angle between its Burgers vector and line direction was smaller than one degree. 


\begin{tabular}{|c|c|}
\hline Property & Value \\
\hline$a_{0}$ & (Davey, 1925)3.61 $\AA$ \\
$b$ & (Kittel and McEuen, 2018) $a_{0} / \sqrt{2}$ \\
$d$ & (Kittel and McEuen, 2018) $a_{0} / \sqrt{3}$ \\
$\gamma$ & (Bonneville and Escaig, 1979)42 $\mathrm{mJ} / \mathrm{m}^{2}$ \\
$\nu$ & (Ledbetter and Naimon, 1974)0.324 \\
$\mu$ & (Schmauder and Mishnaevsky, 2008) $54.6 \mathrm{GPa}$ \\
$T_{m}$ & (Mangum et al., 2001)1358 K \\
$B$ & (Philibert, 1979) $1.5 \times 10^{-5} \mathrm{~Pa}-\mathrm{s}$ \\
\hline
\end{tabular}

Table 1: Parameter values used for face-centered cubic (FCC) $\mathrm{Cu}$; $a_{0}$ is the lattice parameter, $b$ the magnitude of the Burgers vector, $d$ the interplanar separation, $\gamma$ the intrinsic stacking-fault energy, $\nu$ the Poisson's ratio, $\mu$ the shear modulus, $T_{m}$ is the melting temperature and $B$ the viscous drag coefficient at room temperature.

\begin{tabular}{|c|c|c|c|c|}
\hline$h[\AA]$ & $L_{i}[\AA]$ & $y_{i}[\AA]$ & $y_{c}[\AA]$ & $\delta t$ \\
\hline $6 d \approx 12.5$ & $2000 b \approx 5105.3$ & $10 b \approx 25.5$ & 4.4 & 0.0001 \\
$25 d \approx 52.1$ & $350 b \approx 893.4$ & $30 b \approx 76.6$ & 18.5 & 0.01 \\
\hline
\end{tabular}

Table 2: Dipole dimensions in the DD simulations; $h$ is the dipole height, $L_{i}$ the initial dislocation length, $y_{i}$ the initial separation of the dislocations in the direction of $\hat{y}, y_{c}$ their separation at the common cross-slip plane and $\delta t$ the time step.

For each screw chain, a random number $R$ between 0 and 1 was generated. If the cross-slip probability $P$ was larger than $R$, the glide plane of the corresponding screw segment was changed to its deviated plane. Else, the system remained unchanged.

In order to calculate the activation enthalpy and the nucleation length, $\sigma_{S}^{g}, \sigma_{E}^{g}, \sigma_{S}^{c s}$ and $\sigma_{E}^{c s}$ must be obtained as functions of the total stress tensor components. Therefore, the Cartesian coordinate system was rotated such that the [111] direction matched with the $\hat{z}$ axis and the Burgers vector [110] with the $\hat{x}$ axis. The resulting equations of the stress components were given by:

$$
\sigma_{S}^{g}=\sigma_{x z}
$$




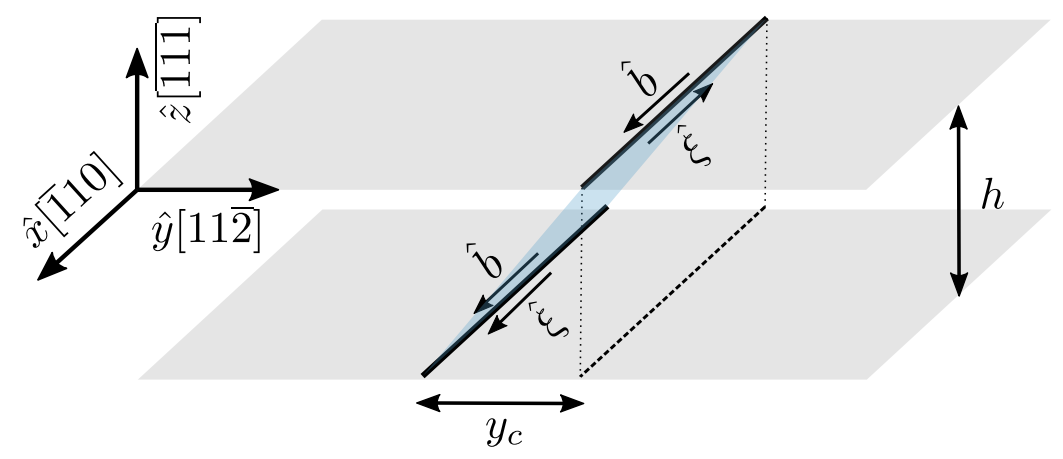

Figure 4: Screw dipole of height $h$ contained in the glide plane (shaded in gray). The dislocation separation at the common cross-slip plane (shaded in blue) is denoted as $y_{c}$.

$$
\begin{gathered}
\sigma_{E}^{g}=\sigma_{y z} \\
\sigma_{S}^{c s}=\frac{2 \sqrt{2} \sigma_{x y}-\sigma_{x z}}{3} \\
\sigma_{E}^{c s}=\frac{7 \sigma_{z y}+2 \sqrt{2}\left(\sigma_{y y}-\sigma_{z z}\right)}{9}
\end{gathered}
$$

The cross-slip mechanism has been extensively studied using atomistic simulations. In particular, Vegge et al. (2000) and Oren et al. (2017) have investigated the kinetics of cross-slip in FCC copper. Both studies offer a quantitative analysis of the activation enthalpy and annihilation rate of screw dipoles, which can be used as a standard to compare against other results. For the purpose of comparison, similar dipole dimensions to theirs were used in the present DD simulations of FCC copper.

Consider a screw dipole of height $h$ with both dislocations arrested in the glide plane, at the intersection with the common cross-slip plane. In FCC structures, the angle between two conjugate planes is $\theta_{c}=\arccos \left(\frac{1}{3}\right) \approx$ $70.5^{\circ}$. Consequently, the dislocation separation in the direction of $\hat{y}$ is $y_{c}=$ $h \cot \theta_{c}$ (see Fig. 4). If under these conditions one of the dislocations crossslips, it would start gliding in the conjugate plane and both dislocations would annihilate each other. For that reason, the activation enthalpy and annihilation rate were calculated at the intersection with the common crossslip plane. 


\begin{tabular}{|c|c|c|c|c|}
\hline$\sigma_{E}^{g}[\mathrm{MPa}]$ & $\Delta H[\mathrm{eV}]$ & $L[\AA]$ & $L_{n}[\AA]$ & $\Gamma_{H T S T}^{0}[\mathrm{~Hz}]$ \\
\hline 0 & 1.190 & 4862 & 32.9 & $3.93 \times 10^{17}$ \\
1300 & 0.295 & 4862 & 10.0 & $6.07 \times 10^{14}$ \\
\hline
\end{tabular}

Table 3: Parameter values obtained from DD simulations for the 13 - $\mathrm{A}$-high dipole; $\sigma_{E}^{g}$ is the compressive Escaig stress applied on the glide plane of the screw dipole, $\Delta H$ the activation enthalpy, $L$ the screw-segment length, $L_{n}$ the nucleation length and $\Gamma_{H T S T}^{0}=\nu \frac{L}{L_{n}} \exp \left(\frac{\Delta H}{k_{b} T_{m}}\right)$ the constant prefactor of Eq. 13.

The dipole dimensions, physical properties and elastic constants used in the DD simulations are shown in Tables 1 and 2.

\section{Results and discussion}

\subsection{Screw dipole of height $1.3 \mathrm{~nm}$}

The DD simulations were performed using non-dissociated dislocations. In consequence, there were no internal Escaig stresses induced by the screw dipole in the present DD simulations. According to linear elasticity theory, a screw dislocation with Burgers vector $\vec{b}=b \hat{x}$ generates non-zero stress components $\sigma_{x y}$ and $\sigma_{x z}$ (Anderson et al., 2017). As a result, a perfect screw dipole can only produce Schmid stresses on the glide and cross-slip planes (see Eqs. 27 and 29). However, a dissociated screw dipole could create both Schmid and Escaig stresses on the glide and cross-slip planes. The screw component of the Shockley partials would induce Schmid stresses on the glide and cross-slip planes as the perfect screw dipole. On the other hand, the edge components of the Shockley partials would introduce Escaig stresses on the glide and cross-slip planes. It can be shown that an edge dislocation with line direction $\hat{\xi}=\hat{x}$ and Burgers vector $\vec{b}=b \hat{y}$ generates non-zero stress components $\sigma_{x x}, \sigma_{y y}, \sigma_{z z}$ and $\sigma_{y z}$ (Anderson et al., 2017). For that reason, the edge component of the Shockley partials would introduce Escaig stresses on the glide and cross-slip planes of a dissociated screw dipole (see Eqs. 28 and 30).

Fig. 5 shows the Arrhenius of the cross-slip rate for the 13 - $\AA$-high dipole. The logarithm of the annihilation rates obtained by Vegge et al. (2000) using MD simulations are also plotted for reference. Since their data points could be fitted well with a straight line, they proposed the following rate equation: 


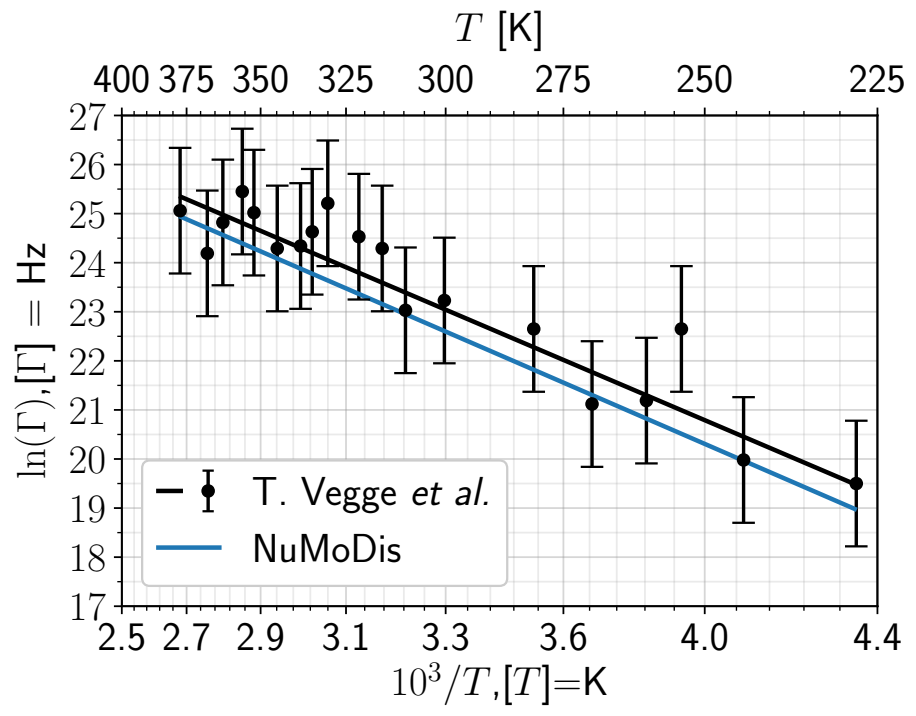

Figure 5: Arrhenius plots of the cross-slip rate for the 13 - $\AA$-high dipole. The black dots with error bars represent the 19 data points obtained by Vegge et al. (2000) using MD simulations. The black line corresponds to the linear fit proposed by Vegge et al. (2000) to model their atomistic data. The blue line represents the model of Esteban-Manzanares et al. (2020) used in the DD simulations. 


\begin{tabular}{|c|c|c|c|c|}
\hline$\sigma_{E}^{g}[\mathrm{MPa}]$ & $\Delta H[\mathrm{eV}]$ & $L[\AA]$ & $L_{n}[\AA]$ & $\Gamma_{H T S T}^{0}[\mathrm{~Hz}]$ \\
\hline 0 & 1.55 & 447 & 32.9 & $8.14 \times 10^{17}$ \\
700 & 0.56 & 447 & 14.8 & $3.61 \times 10^{14}$ \\
900 & 0.45 & 447 & 12.8 & $1.58 \times 10^{14}$ \\
1100 & 0.36 & 447 & 11.2 & $8.86 \times 10^{13}$ \\
1500 & 0.26 & 447 & 9.1 & $4.64 \times 10^{13}$ \\
\hline
\end{tabular}

Table 4: Parameter values obtained from DD simulations for the 50 - $\AA$-high dipole.

$$
\Gamma=\frac{1}{\tau}=\Gamma_{0} \exp \left(-\frac{\Delta H}{k_{b} T}\right)
$$

where $\tau$ is the average annihilation time and $\Gamma_{0}$ is a the rate prefactor. The least squares fit lead them to the values $\Delta H=291 \pm 27 \mathrm{meV}$ and $\Gamma_{0}=7.8 \times 10^{14} \mathrm{~Hz}$.

In order to compensate for the absence of internal Escaig stresses in the DD simulations, a compressive Escaig stress of $1300 \mathrm{MPa}$ was applied on the glide plane. As a result, the activation enthalpy decreased to $\Delta H=0.295$ $\mathrm{eV}$.

The parameter values obtained from DD simulations are shown in table 3. The rate-prefactor value calculated using the model of Esteban-Manzanares et al. (2020) was similar to the one proposed by Vegge et al. (2000) to fit their atomistic data $\left(6.07 \times 10^{14}\right.$ and $7.8 \times 10^{14} \mathrm{~Hz}$, respectively). Therefore, the cross-slip rates were in good quantitative agreement as observed in Fig. 5. It is worth mentioning that the external Escaig stress only affects the dissociation width. For that reason, the screw-segment length remains unaffected by the applied Escaig stress for a given dipole height (see the third column of Table 3).

\subsection{Screw dipole of height $5 \mathrm{~nm}$}

Fig. 6 shows the activation enthalpy of the 50 - $\AA$-high dipole as function of compressive Escaig stress applied on the glide plane. The atomistic results obtained by Oren et al. (2017) using MD simulations are also plotted for reference. The results were in good quantitative agreement. The activation enthalpy at $1500 \mathrm{MPa}$ was approximately $0.3 \mathrm{eV}$ using both simulation techniques. Although not all the values obtained in this work lie within their 


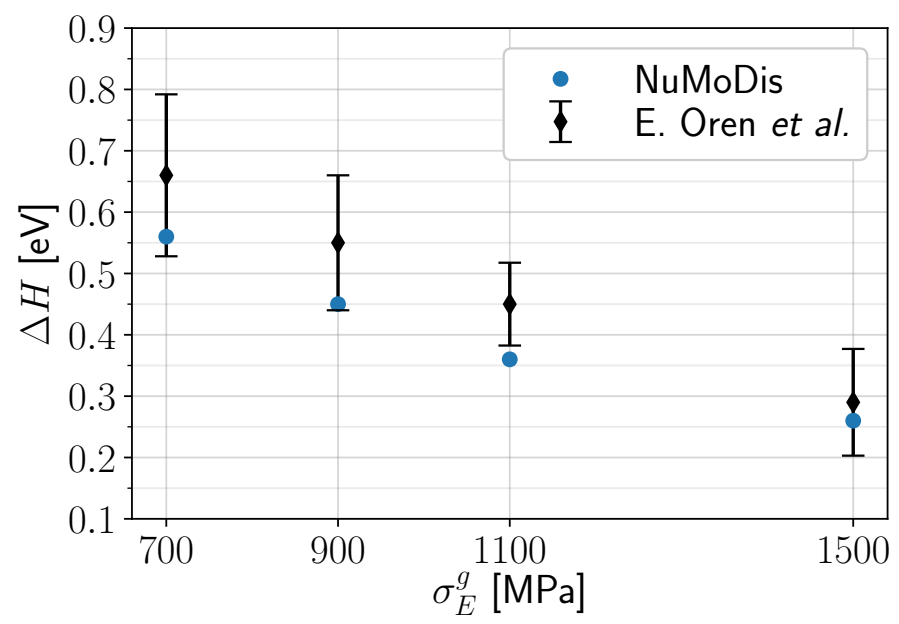

Figure 6: Activation enthalpy of the 50- $\AA$-high dipole as function of compressive Escaig stress applied on the glide plane. The blue circles are the activation enthalpies obtained from the DD simulations using the model of Malka-Markovitz and Mordehai (2019). The black diamonds are the data points obtained by Oren et al. (2017) using MD simulations.

uncertainty, the largest discrepancy was only a small fraction of an electronvolt.

Oren et al. (2017) deduced an energy barrier of $1.05 \pm 15 \% \mathrm{eV}$, which lies within the experimental value of $1.15 \pm 0.37 \mathrm{eV}$ measured by Bonneville et al. (1988). They inferred this value from their data by double interpolation to both zero applied stress and infinite dipole height. On the other hand, the energy barrier of $1.9 \mathrm{eV}$ used in this work was almost the double of their estimate. In spite of it, the simulation results are in good agreement because the activation enthalpy does not decrease linearly with the applied stress. Instead, the activation energy reduces to almost half of its value at $250 \mathrm{MPa}$ (Longsworth and Fivel, 2021), but decreases only $0.3 \mathrm{eV}$ when increasing the stress magnitude from 700 to $1500 \mathrm{MPa}$ (see table 4).

Fig. 7 shows the Arrhenius plots of the cross-slip rate for the 50 - $\AA$-high dipole as function of compressive Escaig stress applied on the glide plane. Most values were in good quantitative agreement. The harmonic approximation used in the rate equation assumes that the transition state is close to the original one. This is most accurate at sufficiently low temperatures, when the transition cannot be induced by thermal activation. In the investigated 


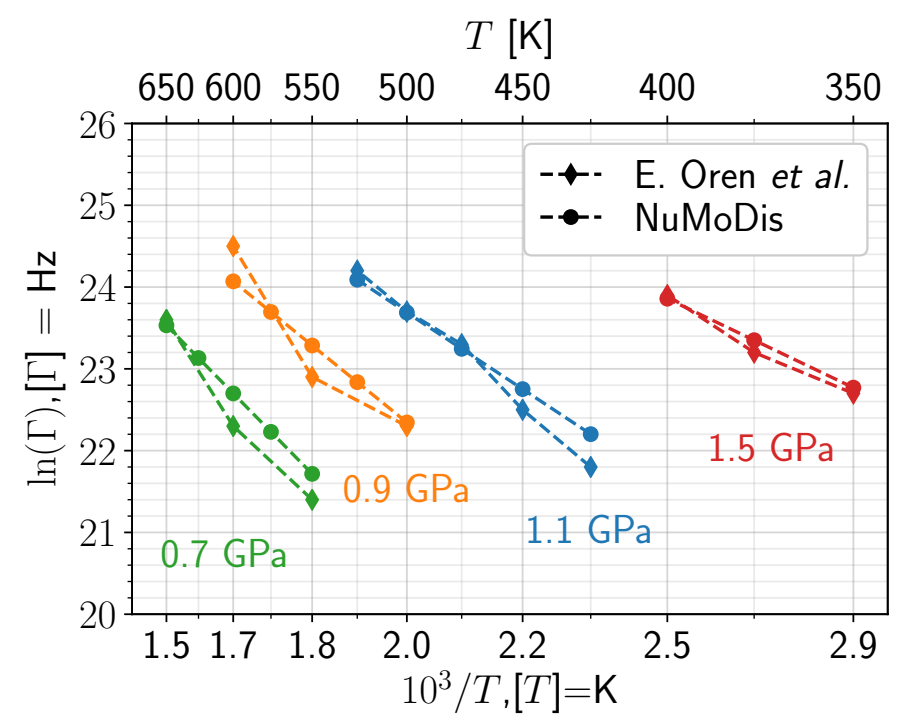

Figure 7: Arrhenius plots of the cross-slip rate for the 50 - $\AA$-high dipole as function of compressive Escaig stress applied on the glide plane. The dots are the cross-slip rates obtained from the DD simulations using the model of Esteban-Manzanares et al. (2020). The diamonds correspond to the atomistic results of Oren et al. (2017). The temperature range goes from $350 \mathrm{~K}$ to $650 \mathrm{~K}$ in steps of $25 \mathrm{~K}$. 
temperature range, the activation enthalpy is larger than the thermal energy. For that reason, the cross-slip rates calculated using the HTST should be valid.

In faulted screw dipoles, the compressive Escaig stresses in the glide plane are expected to increase with decreasing dipole height (Rasmussen et al., 2000). As a result, the nucleation length modeled using Eq. 21 would decrease with decreasing dipole height. However, there are no internal Escaig stresses in perfect screw dipoles. In consequence, the nucleation length was $32.9 \AA$ at zero applied stress, independently of the dipole height (compare Tables 3 and 4). As reference, the nucleation length of aluminum was approximately $28 \AA$ according to Esteban-Manzanares et al. (2020).

\section{Summary and conclusion}

The objective of this work is to propose a method to compute the crossslip rate of screw segments in DD simulations without relying on scaling factors, yet able to reproduce atomistic results. Thus, all the input information used to compute the cross-slip rate was retrieved from DD simulations. The stress and forces on the dislocation segments were calculated using the analytical expressions obtained by Cai et al. (2006), who developed the nonsingular theory of dislocations. As a consequence, the core-width parameter introduced to obtain those non-singular expressions influenced the DD simulations strongly. In particular, atomistic and continuum energies are only comparable if the core-width value is deduced using both an atomistic and a continuum dislocation model. Since the aim was to compare the DD simulations' outcome with previous atomistic results, the core-width value was calculated using the theory of Schöck (2010) as proposed by Ramírez et al. (2012). They deduced an expression for the core-width parameter based on both atomistic and continuum dislocation models.

The cross-slip rate of screw segments was calculated using the atomisticbased model proposed by Esteban-Manzanares et al. (2020) (see Eq. 13), which combines the HTST framework and the NM rule. In that model, the only two input parameters that depend on the local stress are the activation enthalpy and the nucleation length. Both quantities were obtained from the LT model of cross-slip as follows.

The activation enthalpy was obtained by evaluating the cross-slip energy $H(\sigma, l)$ (see Eq. 24) at the critical length satisfying Eq. 25 . In the model of Malka-Markovitz and Mordehai (2019), the activation enthalpy is a function 
of the microstructure parameters and the stress components acting on the crystallographic planes, which are easily accessible from the DD simulation. With the aim of calibrating their model, the unstressed energy barrier was set to $1.9 \mathrm{eV}$ as obtained with the DD simulations of FCC copper (Ramírez et al., 2012; Longsworth and Fivel, 2021).

The nucleation length was inferred from the LT model of cross-slip developed by Malka-Markovitz and Mordehai (2018) (see Eq. 21). It is expressed as a function of the line tension, the microstructure parameters and the Escaig stress on the glide plane. In said model, the only free variable is the line tension, which is proportional to the line-energy parameter (see Eq. 14). Typical values of the line-energy parameter are in the range between 0.1 and 0.6 (Kang et al., 2014). For that reason, an average line-energy value of 0.3 was used in the present work.

In summary, the cross-slip modeling proposed in this paper by combining equations 13, 21 and 24 with $\alpha=0.3$ and $\Delta E_{0}=1.9 \mathrm{eV}$ can be reliably used in DD simulations of FCC copper. The activation enthalpies and cross-slip rates obtained were in good quantitative agreement with the atomistic results of Oren et al. (2017) and Vegge et al. (2000). These results ratify that the HTST approximation and the MN rule are valid in the studied temperature range and stress conditions.

\section{Acknowledgments}

The authors acknowledge use of the 3D dislocation dynamics code $\mathrm{Nu}-$ MoDis jointly developed at CEA (L. Dupuy) and Univ. Paris XII (M. Bletry). This work has received funding from the European Research Council (ERC) under the European Union's Horizon 2020 research and innovation programme (ERC-2016-COG grant agreement number 725142).

\section{References}

P. Anderson, J. Hirth, and J. Lothe. Theory of Dislocations. Cambridge University Press, 2017. ISBN 9780521864367. URL https://books .google. $\mathrm{fr} /$ books? id=LK7DDQAAQBAJ.

J. Bonneville and B. Escaig. Cross-slipping process and the stress-orientation dependence in pure copper. Acta Metallurgica, 27(9):1477-1486, 1979. ISSN 00016160. doi: 10.1016/0001-6160(79)90170-6. URL https:// linkinghub.elsevier.com/retrieve/pii/0001616079901706. 
J. Bonneville, B. Escaig, and J. Martin. A study of cross-slip activation parameters in pure copper. Acta Metallurgica, 36(8):1989-2002, 1988. ISSN 00016160. doi: 10.1016/0001-6160(88)90301-X. URL https : //linkinghub.elsevier.com/retrieve/pii/000161608890301X.

W. Cai, A. Arsenlis, C. Weinberger, and V. Bulatov. A non-singular continuum theory of dislocations. Journal of the Mechanics and Physics of Solids, 54(3):561-587, 2006. ISSN 00225096. doi: 10.1016/j.jmps. 2005.09.005. URL https://linkinghub.elsevier.com/retrieve/pii/ S002250960500195X.

J. Chaussidon, C. Robertson, D. Rodney, and M. Fivel. Dislocation dynamics simulations of plasticity in Fe laths at low temperature. Acta Materialia, 56 (19):5466-5476, 2008. ISSN 13596454. doi: 10.1016/j.actamat.2008.07.047. URL http://dx.doi.org/10.1016/j . actamat.2008.07.047.

W. Davey. Precision Measurements of the Lattice Constants of Twelve Common Metals. Physical Review, 25(6):753-761, 1925. ISSN 0031899X. doi: 10.1103/PhysRev.25.753. URL https://link.aps.org/doi/ 10.1103/PhysRev.25.753.

C. Déprés, C. Robertson, and M. Fivel. Low-strain fatigue in AISI 316L steel surface grains: a three-dimensional discrete dislocation dynamics modelling of the early cycles I. Dislocation microstructures and mechanical behaviour. Philosophical Magazine, 84(22):2257-2275, 2004. ISSN 1478-6435. doi: 10.1080/14786430410001690051. URL http://www.tandfonline. com/doi/abs/10.1080/14786430410001690051.

J. Drouet, L. Dupuy, F. Onimus, F. Mompiou, S. Perusin, and A. Ambard. Dislocation dynamics simulations of interactions between gliding dislocations and radiation induced prismatic loops in zirconium. Journal of Nuclear Materials, 449(1-3):252-262, jun 2014. ISSN 00223115. doi: 10.1016/j.jnucmat.2013.11.049. URL http://dx.doi.org/10.1016/j.jnucmat.2013.11.049https: //linkinghub.elsevier. com/retrieve/pii/S0022311513012877.

G. Esteban-Manzanares, R. Santos-Güemes, I. Papadimitriou, E. Martínez, and J. LLorca. Influence of the stress state on the cross-slip free energy barrier in Al: An atomistic investigation. Acta Materialia, 184:109-119, 
2020. ISSN 13596454. doi: 10.1016/j.actamat.2019.10.055. URL https: //doi.org/10.1016/j.actamat.2019.10.055.

A. V. Granato, K. Lücke, J. Schlipf, and L. J. Teutonico. Entropy Factors for Thermally Activated Unpinning of Dislocations. Journal of Applied Physics, 35(9):2732-2745, 1964. ISSN 0021-8979. doi: 10.1063/1.1713833. URL http://aip.scitation.org/doi/10.1063/1.1713833.

P. Hirsch. The Physics of Metals. Cambridge University Press, 2009. ISBN 9780521113106. URL https://books.google.fr/books?id= 5JOKPwAACAAJ.

A. Hussein, S. Rao, M. Uchic, D. Dimiduk, and J. El-Awady. Microstructurally based cross-slip mechanisms and their effects on dislocation microstructure evolution in fcc crystals. Acta Materialia, 85:180-190, 2015. ISSN 13596454. doi: 10.1016/j.actamat.2014.10.067. URL http://dx. doi.org/10.1016/j. actamat.2014.10.067.

S. Ikeno and E. Furubayashi. Behavior of dislocations in niobium under stress. Physica Status Solidi (a), 12(2):611-622, 1972. ISSN 00318965. doi: 10.1002/pssa.2210120234. URL http://doi.wiley.com/10.1002/ pssa. 2210120234 .

P. Jackson. Dislocation modelling of shear in f.c.c. crystals. Progress in Materials Science, 29(1-2):139-175, 1985. ISSN 00796425. doi: 10. 1016/0079-6425(85)90009-X. URL https://linkinghub. elsevier.com/ retrieve/pii/007964258590009X.

W. Johnston and J. Gilman. Dislocation Multiplication in Lithium Fluoride Crystals. Journal of Applied Physics, 31(4):632-643, 1960. ISSN 00218979. doi: 10.1063/1.1735655. URL http://aip.scitation.org/doi/ $10.1063 / 1.1735655$.

K. Kang, J. Yin, and W. Cai. Stress dependence of cross slip energy barrier for face-centered cubic nickel. Journal of the Mechanics and Physics of Solids, 62:181-193, 2014. ISSN 00225096. doi: 10.1016/j.jmps. 2013.09.023. URL https://linkinghub.elsevier.com/retrieve/pii/ S002250961300207X. 
C. Kittel and P. McEuen. Introduction to Solid State Physics. John Wiley \& Sons, 2018. ISBN 9781119454168. URL https://books.google.fr/ books?id=S3gAugEACAAJ.

L. Kubin, G. Canova, M. Condat, B. Devincre, V. Pontikis, and Y. Bréchet. Dislocation Microstructures and Plastic Flow: A 3D Simulation. Solid State Phenomena, 23-24:455-472, 1992. ISSN 1662-9779. doi: 10.4028/ www.scientific.net/SSP.23-24.455. URL https://www.scientific.net/ SSP. 23-24.455.

W. Kuykendall, Y. Wang, and W. Cai. Stress effects on the energy barrier and mechanisms of cross-slip in FCC nickel. Journal of the Mechanics and Physics of Solids, 144:1-13, 2020. ISSN 00225096. doi: 10.1016/j.jmps. 2020.104105. URL https://doi.org/10.1016/j.jmps.2020.104105.

H. Ledbetter and E. Naimon. Elastic Properties of Metals and Alloys. II. Copper. Journal of Physical and Chemical Reference Data, 3(4): 897-935, 1974. ISSN 0047-2689. doi: 10.1063/1.3253150. URL http: //aip.scitation.org/doi/10.1063/1.3253150.

H. Liu, X. Zhou, D. Hu, J. Song, R. Wang, and J. Mao. A new variational line tension model for accurate evaluation of the stress effect on cross-slip energy barrier in face-centered cubic metals. Scripta Materialia, 166:2428, 2019. ISSN 13596462. doi: 10.1016/j.scriptamat.2019.03.002. URL https://doi.org/10.1016/j.scriptamat.2019.03.002.

M. Longsworth and M. Fivel. The effect of stress on the cross-slip energy in face-centered cubic metals: A study using dislocation dynamics simulations and line tension models. Journal of the Mechanics and Physics of Solids, 148:104281, 2021. ISSN 00225096. doi: 10.1016/j.jmps.2020. 104281. URL https://doi.org/10.1016/j.jmps.2020.104281https: //linkinghub.elsevier.com/retrieve/pii/S0022509620304865.

A. Malka-Markovitz and D. Mordehai. Cross-slip in face-centered cubic metals: a general Escaig stress-dependent activation energy line tension model. Philosophical Magazine, 98(5):347-370, 2018. doi: 10.1080/14786435.2017. 1406194. URL https://doi.org/10.1080/14786435.2017.1406194.

A. Malka-Markovitz and D. Mordehai. Cross-slip in face centred cubic metals: a general full stress-field dependent activation energy line-tension model. 
Philosophical Magazine, 99(12):1460-1480, 2019. ISSN 1478-6435. doi: 10.1080/14786435.2019.1584410. URL https://www.tandfonline.com/ doi/full/10.1080/14786435.2019.1584410.

A. Malka-Markovitz, B. Devincre, and D. Mordehai. A molecular dynamics-informed probabilistic cross-slip model in discrete dislocation dynamics. Scripta Materialia, 190:7-11, 2021. ISSN 13596462. doi: 10.1016/j.scriptamat.2020.08.008. URL https://doi.org/10.1016/j. scriptamat.2020.08.008.

B. W. Mangum, G. T. Furukawa, K. G. Kreider, C. W. Meyer, D. C. Ripple, G. F. Strouse, W. L. Tew, M. R. Moldover, B. C. Johnson, H. W. Yoon, C. E. Gibson, and R. D. Saunders. The Kelvin and temperature measurements. Journal of Research of the National Institute of Standards and Technology, 106(1):105, 2001. doi: 10.6028/jres.106.006. URL https://nvlpubs.nist.gov/nistpubs/jres/106/1/j61man.pdf.

W. Meyer and H. Neldel. Relation between the energy constant and the quantity constant in the conductivity-temperature formula of oxide semiconductors. Z. tech. Phys, 18(12):588-593, 1937.

E. Oren, E. Yahel, and G. Makov. Kinetics of dislocation cross-slip: A molecular dynamics study. Computational Materials Science, 138:246254, 2017. ISSN 09270256. doi: 10.1016/j.commatsci.2017.06.039. URL http://dx.doi.org/10.1016/j.commatsci.2017.06.039.

J. Philibert. Dislocations et déformation plastique. Les éditions de la physique in École d'été d'Yravals, 101, 1979.

B. Ramírez, N. Ghoniem, and G. Po. Ab initio continuum model for the influence of local stress on cross-slip of screw dislocations in fcc metals. Physical Review B, 86(9):1-11, 2012. ISSN 1098-0121. doi: 10. 1103/PhysRevB.86.094115. URL https://link.aps.org/doi/10.1103/ PhysRevB.86.094115.

T. Rasmussen, T. Vegge, T. Leffers, O. Pedersen, and K. Jacobsen. Simulation of structure and annihilation of screw dislocation dipoles. Philosophical Magazine A: Physics of Condensed Matter, Structure, Defects and Mechanical Properties, 80(5):1273-1290, 2000. ISSN 01418610. doi: $10.1080 / 01418610008212115$. 
S. Schmauder and L. Mishnaevsky. Micromechanics and Nanosimulation of Metals and Composites: Advanced Methods and Theoretical Concepts. Springer Berlin Heidelberg, 2008. ISBN 9783540786788. URL https: //books.google.fr/books?id=QcyxI-3EG4cC.

G. Schöck. Atomic dislocation core parameters. physica status solidi (b), 247 (2):265-268, 2010. ISSN 03701972. doi: 10.1002/pssb.200945379. URL http://doi.wiley.com/10.1002/pssb. 200945379.

C. Sobie, L. Capolungo, D. McDowell, and E. Martínez. Modal analysis of dislocation vibration and reaction attempt frequency. Acta Materialia, 134:203-210, 2017. ISSN 13596454. doi: 10.1016/j.actamat.2017.02.005. URL http://dx.doi.org/10.1016/j.actamat.2017.02.005.

A. Stroh. Constrictions and Jogs in Extended Dislocations. Proceedings of the Physical Society. Section B, 67(5):427-436, 1954. ISSN 0370-1301. doi: 10.1088/0370-1301/67/5/307. URL https://iopscience.iop.org/ article/10.1088/0370-1301/67/5/307.

M. Sudmanns, M. Stricker, D. Weygand, T. Hochrainer, and K. Schulz. Dislocation multiplication by cross-slip and glissile reaction in a dislocation based continuum formulation of crystal plasticity. Journal of the Mechanics and Physics of Solids, 132:1-19, 2019. ISSN 00225096. doi: 10.1016/j.jmps.2019.103695. URL https://linkinghub.elsevier.com/ retrieve/pii/S0022509619306428.

T. Vegge, T. Rasmussen, T. Leffers, O. B. Pedersen, and K. W. Jacobsen. Determination of the of Rate Cross Slip of Screw Dislocations. Physical Review Letters, 85(18):3866-3869, 2000. ISSN 0031-9007. doi: 10.1103/PhysRevLett.85.3866. URL https://link.aps.org/doi/ 10.1103/PhysRevLett.85.3866.

M. Verdier, M. Fivel, and I. Groma. Mesoscopic scale simulation of dislocation dynamics in fcc metals: Principles and applications. Modelling and Simulation in Materials Science and Engineering, 6:755-770, 1998. ISSN 0965-0393. doi: 10.1088/0965-0393/6/6/007. URL https: //iopscience.iop.org/article/10.1088/0965-0393/6/6/007.

G. H. Vineyard. Frequency factors and isotope effects in solid state rate processes. Journal of Physics and Chemistry of Solids, 3(1-2):121-127, 
1957. ISSN 00223697. doi: 10.1016/0022-3697(57)90059-8. URL https : //linkinghub.elsevier.com/retrieve/pii/0022369757900598.

D. Weygand, L. Friedman, E. Giessen, and A. Needleman. Aspects of boundary-value problem solutions with three-dimensional dislocation dynamics. Modelling and Simulation in Materials Science and Engineering, 10(4):437-468, 2002. ISSN 09650393. doi: 10.1088/0965-0393/10/4/306. URL https://iopscience.iop.org/article/10.1088/0965-0393/10/ $4 / 306$.

H. Zbib, M. Rhee, and J. Hirth. On plastic deformation and the dynamics of 3D dislocations. International Journal of Mechanical Sciences, 40(2-3):113-127, 1998. ISSN 00207403. doi: 10.1016/S0020-7403(97) 00043-X. URL https://linkinghub.elsevier.com/retrieve/pii/ S002074039700043X. 